\title{
ON NULLITY DISTRIBUTIONS
}

\author{
B Y \\ SIN-LENG TAN
}

\begin{abstract}
The nullity concept of Riemannian manifolds is extended to affine manifolds. Results obtained by Chern and Kuiper and Maltz on Riemannian manifolds are generalized to affine manifolds. A structure theorem for affine symmetric spaces is obtained. Finally, the nullity concept is generalized to study the partial integrability of certain geometric structures.
\end{abstract}

1. Introduction. In [1], Chern and Kuiper define a distribution on a Riemannian manifold $M$ which assigns to each point $m \in M$, the subspace

$$
N_{m}=\left\{X \in T M_{m} \mid R(X, Y)=0 \text { for all } Y \in T M_{m}\right\}
$$

where $R$ is the curvature of the Riemannian connection on $M$. It is called the nullity space at $m$. The distribution defined by the subspace $N_{m}$ at each point $m$ of $M$ is called the nullity distribution $N$ of the Riemannian manifold $M$. Set $\mu(m)$ $=$ dimension $N_{m}$. The number $\mu(m)$ is called the index of nullity at $m$. Chern and Kuiper showed that if $\mu(m)$ is constant in a neighborhood then $N$ constitutes a completely integrable distribution there, and that the leaves of the resulting foliation are flat. Later, Maltz [5] showed that the leaves are also totally geodesic.

In $\S \S 2$ and 3 , we extend the definition of nullity to affine manifolds and show that the resulting nullity distribution is still integrable. We show also that the leaves are totally geodesic and flat with respect to an induced connection on the leaf if the curvature tensor is parallel. In $\S 4$, we investigate the nullity distribution on symmetric spaces and obtain a structure theorem (Theorem 4.6) for affine symmetric spaces. This type of structure theorem is well known for simply connected Riemannian symmetric spaces ( $\$ 5)$. In $\S 7$, distributions generated by isometric actions of a compact Lie group are discussed. It is shown that such Lie group actions define a metric connection on the manifold with nullity index at least the dimension of the Lie group. Finally in §8, we observe that the study of nullity distributions is indeed a study of partial integrability of the underlying geometric structures. Thus, we can extend this concept to study the nullity

Received by the editors July 10, 1975 . $53 \mathrm{C} 10$.

AMS (MOS) subject classifications (1970). Primary 53C05, 58A30, 53C35; Secondary

Key words and phrases. Affine manifolds, nullity distributions, symmetric spaces, partial integrability. 
distributions of certain type of tensors which characterize the underlying geometric structures.

This paper was motivated by the following problem. If the ordinary characteristic ring Pont* $(M)$ of the manifold $M$ fails to give much information about the manifold $M$, how do we produce a set of secondary invariants such as to supplement the ordinary characteristic ring? By using the generalized characteristic map constructed by Kamber and Tondeur [3], the author was able to produce such a set of secondary invariants [8].

The author would like to express his gratitude to his advisor, Professor F. Kamber, for his encouragement and forbearance during this work. The results in this paper are contained in the author's thesis [8].

2. Nullity distribution on affine manifolds. Let $(M, \nabla)$ be an affine manifold, namely a manifold $M$ on which $\nabla$ is the connection on the tangent bundle $T M$. Let $R$ and $T$ be the curvature and torsion tensors of $\nabla$. The curvature tensor $R$ can be considered as a bundle map $R: T M \rightarrow \operatorname{Hom}(T M, \operatorname{End}(T M))$. The nullity distribution $N$ of the affine manifold $(M, \nabla)$ can be defined as the kernel of the map $R$. The nullity space at a point $m$ of $M$ is the subspace $N_{m}$ which is equal to $\operatorname{Ker} R_{m}$. The following proposition is similar to those in [5].

Proposition 2.1. (i) The function $\mu(m)$ is upper semicontinuous.

(ii) The set $V$ where $\mu(m)$ assumes its minimum value is open in $M$.

Proof. Statement (ii) follows from (i). Statement (i) follows from the fact that $N$ is the kernel of a bundle map, and the dimension at each fibre is always upper semicontinuous. Q.E.D.

The above proposition says that the distribution $N$ is nonsingular only on the set $V$. In general $N$ is singular. The index $\mu$ at any point will not take the value $n-1$, where $n$ is the dimension of the manifold $M$. Since if $\mu(m)>n-1$, then there exist linearly independent vectors $X_{1}, X_{2}, \ldots, X_{n-1}$ in $T M_{m}$ such that $X_{i} \in N_{m}$, i.e., $R\left(X_{i},-\right)=0$, for $i=1,2, \ldots, n-1$. Take $X_{n} \in T M_{m}$ so that $X_{1}, X_{2}, \ldots, X_{n}$ form a basis in $T M_{m}$. Since $R\left(X_{i}, X_{n}\right)=0$ for $i=1,2$, $\ldots, n-1$, it follows that $R\left(X_{n},-\right)=0$. Hence $\mu(m)=n$. Consequently, if $N$ is nonsingular, then $N$ cannot have codimension 1.

Considering a possible singular nullity distribution $N$ on $M$, we say that $N$ is involutive if and only if the presheaf of cross sections over open sets of $M$ is involutive.

THEOREM 2.2. The distribution $N$ is involutive.

Proof. Let $U$ be an open set, and $Y, Z \in \Gamma(U, N)$. It is sufficient to show that $R([Y, Z], X)=0$ for all $X \in \Gamma(U, N)$. Since 


$$
\nabla_{X}(R(Y, Z))=\nabla_{Y}(R(Z, X))=\nabla_{Z}(R(X, Y))=0
$$

therefore,

$$
0=S\left(\nabla_{X}(R(Y, Z))\right)=S\left\{\left(\nabla_{X} R\right)(Y, Z)+R\left(\nabla_{X} Y, Z\right)+R\left(Y, \nabla_{X} Z\right)\right\},
$$

where $S$ denotes cyclic sum. Recall the following Bianchi second identity:

$$
S\left\{\left(\nabla_{X} R\right)(Y, Z)+R(T(X, Y), Z)\right\}=0 .
$$

Therefore, when we substitute $\left(\nabla_{X} R\right)(Y, Z)$, we have

$$
0=S\left\{-R(T(X, Y), Z)+R\left(\nabla_{X} Y, Z\right)+R\left(Y, \nabla_{X} Z\right)\right\}
$$

By actually carrying out the cyclic sum, and noting that $Y, Z \in \Gamma(U, N)$, we obtain

$$
0=-R\left(T(Y, Z)+\nabla_{Y} Z-\nabla_{Z} Y, X\right)
$$

Since $T(Y, Z)=\nabla_{Y} Z-\nabla_{Z} Y-[Y, Z]$, we find that $R([Y, Z], X)=0$. This proves the theorem. Q.E.D.

The following corollary extends a Chern-Kuiper result in [1] to the affine case.

COROLlaRY 2.3. If $\mu(m)$ is locally constant on an open submanifold $K$ of $M$, then $N$ is integrable on $K$.

At this point, unlike the Riemannian case, we cannot conclude that the leaves of $N$ are 'flat' because the affine connection $\nabla$ on $M$ does not induce connections on the leaves.

Let $f: M \rightarrow M^{\prime}$ be a smooth map between two manifolds. We recall that a vector field $X$ on $M$ is $f$-related to a vector field $X^{\prime}$ of $M^{\prime}$ if $f\left(X_{m}\right)=X_{f(m)}^{\prime}$ for all $m \in M$.

Proposition 2.4. Let $f: M \rightarrow M$ be an (local) affine transformation of the affine manifold $(M, \nabla)$. Then $\mu(m)=\mu(f(m))$.

Proof. Since $f$ is a transformation, every vector field $X$ is $f$-related to $f_{*} X$. Since $f$ is an affine transformation, $f_{*} R(X, Y) Z=R\left(f_{*} X, f_{*} Y\right) f_{*} Z$ ( see [4, Proposition 1.2, Chapter VI]). Now the proposition follows immediately because $f_{*}$ is an isomorphism. Q.E.D.

Consequently, if the manifold $M$ is locally affine homogeneous, i.e., the pseudogroup of local affine transformations is locally transitive, then $N$ is a nonsingular distribution.

3. Leaves of nullity distributions. Throughout this section, we assume that the nullity distribution $N$ on an affine manifold $(M, \nabla)$ is nonsingular, and that 
the curvature tensor is parallel with respect to $N$, that is, $\nabla_{X} R=0$ for all $X \in N$. We note that for a Riemannian manifold, the curvature tensor of its canonical connection is already parallel with respect to its nullity distribution.

Let $M^{\prime}$ be a submanifold of $M$. In general, the connection $\nabla$ on $M$ does not induce a connection on $M^{\prime}$. In the case of Riemannian manifold, it is easy to see that the canonical Riemannian connection on $M$ does induce a connection $\nabla^{\prime}$ on the submanifold $M^{\prime}$. But if $M^{\prime}$ is an auto-parallel submanifold of the affine manifold $(M, \nabla)$, that is, for each vector $X \in T M_{m}^{\prime}$, and each curve $\tau$ in $M^{\prime}$ starting from $m$, the parallel displacement of $X$ along $\tau$ (with respect to the affine connection of the ambient space $M$ ) yields a vector tangent to $M^{\prime}$, then the affine connection on $M$ induces a connection of $M^{\prime}$ in a natural manner.

LEMma 3.1. The submanifold $M^{\prime}$ of an affine manifold $(M, \nabla)$ is an autoparallel submanifold if and only if $\nabla_{X} Y$ is tangent to $M^{\prime}$ for all $X, Y \in \mathfrak{X}\left(M^{\prime}\right)$. Hence $\nabla$ induces a connection $\nabla^{\prime}$ on $M^{\prime}$ in a natural manner.

Proof. See [4, §8, Chapter 7].

Proposition 3.2. Every leaf of $N$ is auto-parallel.

Proof. Let $L$ be an integral submanifold of $N$. From the preceding lemma, we need to show that $\nabla_{X} Y$ is tangent to $L$ for $X, Y \in \mathfrak{X}(L)$. It is sufficient to prove the following.

LEMma 3.3. If $X, Y$ are two vector fieids which lie in the nullity distribution $N$, then $\nabla_{X} Y \in \Gamma(N)$.

Proof. We need to show $R\left(\nabla_{X} Y, Z\right)=0$ for all $Z \in \mathfrak{X}(M)$. Since $X, Y \in$ $\Gamma(N)$, we have $\nabla_{X}(R(Y, Z))=0$ and $R\left(Y, \nabla_{X} Z\right)=0$. Therefore,

$$
\begin{aligned}
R\left(\nabla_{X} Y, Z\right) & =-\left(\nabla_{X} R\right)(Y, Z)+\nabla_{X}(R(Y, Z))-R\left(Y, \nabla_{X} Z\right) \\
& =-\left(\nabla_{X} R\right)(Y, Z)=0
\end{aligned}
$$

since $R$ is parallel with respect to $N$. Q.E.D.

Lemma 3.4. Every auto-parallel submanifold $M^{\prime}$ of $M$ is totally geodesic.

Proof. See [4, §8, Chapter 7].

THEOREM 3.5. Every leaf $L$ of $N$ is totally geodesic and flat with respect to the induced connection $\nabla^{\prime}$ on $L$.

PROOF. The proof follows easily from the above lemma and Proposition 3.2.

Theorems 2.2 and 3.5 generalize the theorems of Chern and Kuiper and 
Maltz for Riemannian nullity distributions. It is quite possible that what Maltz did in his thesis could also be generalized to affine cases.

4. Symmetric spaces. We follow the definitions and notations in [4, Chapter XI].

Proposition 4.1. The nullity distribution $N$ of an affine locally symmetric space $(M, \nabla)$ is nonsingular.

Proof. By Proposition 2.4, this amounts to showing that $(M, \nabla)$ is affine locally transitive. But the set of local symmetries is clearly locally affine transitive, and this proves the proposition. Q.E.D.

Consequently, the assumption that $N$ is nonsingular is naturally satisfied on (local) symmetric spaces. The leaves of $N$ are of course flat and totally geodesic submanifolds of $M$ (Theorem 3.5), since the curvature tensor is parallel here.

We shall now assume that $(M, \nabla)$ is connected (globally) affine symmetric. Thus, $(M, \nabla)$ can be identified with the reductive homogeneous space $G / H$ with the canonical $G$-invariant connection $\nabla$, where $G$ is the identity component of the group of affine transformations of $M$, and $H$ is the isotropy subgroup of $G$ at a fixed point $0 \in M$. The symmetries on $M$ induce an involution $\sigma$ on $G$ such that $H$ consists of elements fixed by $\sigma$.

Let $\mathfrak{g}, \mathfrak{h}$ be the Lie algebras of $G, H$ respectively. Since $G / H$ is reductive, the Lie algebra $\mathfrak{g}$ may be decomposed into a vector space direct sum of $\mathfrak{h}$ and an ad $H$-invariant subspace $\mathrm{m}$. Moreover, at $0 \in M$,

$$
R(X, Y) Z=-[[X, Y], Z], \text { for } X, Y, Z \in T M_{0} \text {. }
$$

Here, $T M_{0}$ is identified with $\mathfrak{m}$ in the decomposition $\mathfrak{g}=\mathfrak{h}+\mathfrak{m}$.

Proposition 4.2. Let $\mathrm{m}^{\prime}=N_{0}$. Then, $\mathrm{m}^{\prime}$ is the largest abelian ideal of $\mathfrak{g}$ in $\mathrm{m}$. Moreover, $\mathrm{m}^{\prime}$ satisfies

(i) $\left[\mathrm{m}^{\prime}, \mathrm{m}\right]=0$;

(ii) $\left[\mathfrak{m}^{\prime},[\mathfrak{m}, \mathfrak{m}]\right]=0$.

Proof. We first prove a lemma.

LEMMA 4.3. The isotropy representation of $H$ in $M=G / H$ is faithful.

Proof. We claim that for $h_{1}, h_{2} \in H,\left(h_{1} *\right)_{0}=\left(h_{2} *\right)_{0}$ implies $h_{1}=h_{2}$ on $G / H$. But this fact follows from [4, Lemma 4, p. 254] since $h_{1}, h_{2}$ are $a$ fortiori affine mappings. Clearly, $G$ acts effectively on $M$, therefore $h_{1}=h_{2}$. Q.E.D.

Proof of Proposition. We first prove (i). From the definition of $\mathrm{m}^{\prime}$, we have 


$$
\begin{aligned}
\mathfrak{m}^{\prime} & =\{X \in \operatorname{m} \mid R(X, Y) Z=0 \text { for all } Y, Z \in \mathfrak{m}\} \\
& =\{X \in \operatorname{mlad}[X, Y] Z=0\} .
\end{aligned}
$$

Thus, for $X \in \mathfrak{m}^{\prime}, Y \in \mathfrak{m}$, we have $\operatorname{ad}[X, Y]=0$. Since $[X, Y] \in \mathfrak{h}$ and also $H$ acts faithfully on $m$ (lemma above), we have $[X, Y]=0$. This proves (i).

It follows from (i) that $\mathrm{m}^{\prime}$ is an abelian Lie algebra. To show that $\mathrm{m}^{\prime}$ is an ideal of $\mathfrak{g}$, we need to show that $[X, Y] \in \mathrm{m}^{\prime}$, for $X \in \mathrm{m}^{\prime}, Y \in \mathfrak{h}$. Now

$$
\begin{aligned}
{[X, Y] \in \mathfrak{m}^{\prime} } & \Leftrightarrow[[[X, Y], Z], W]=0 \text { for all } Z, W \in \mathfrak{m} \\
& \Leftrightarrow[[[Y, Z], X], W]-[[[Z, X], Y], W]=0 .
\end{aligned}
$$

Since $[Y, Z] \in m$, therefore by $(i)$, we have $[[Y, Z], X]=0$. Hence, the first term vanishes. Similarly, the second term also vanishes. This proves $\mathrm{m}^{\prime}$ is an abelian ideal of $\boldsymbol{g}$ in $\mathrm{m}$.

Property (ii) can be proved by using (i) and the Jacobi identity. Finally, the fact that $\mathrm{m}^{\prime}$ is the largest abelian ideal of $g$ in $\mathrm{m}$ follows immediately because these properties characterize $m^{\prime}$. Q.E.D.

The following corollary is immediate.

COROllary 4.4. (i) The center $\mathrm{z}$ of $\mathrm{g}$ is contained in $\mathrm{m}^{\prime}$.

(ii) If $[\mathrm{m}, \mathrm{m}]=\mathrm{b}$, then $\mathrm{m}^{\prime}=\mathrm{z}$.

It is now clear that we can prove a structure theorem on symmetric spaces. Let $G^{\prime}$ be the connected normal abelian Lie subgroup of $G$ which has $m^{\prime}$ as its Lie algebra.

LEMMA 4.5. The subgroup $G^{\prime}$ is a closed subgroup of $G$.

Proof. Let $\bar{G}^{\prime}$ be the closure of $G^{\prime}$ and let $\bar{m}$ be its Lie algebra. $G^{\prime}$ is closed if and only if $\bar{m}=m^{\prime}$. Clearly, $\bar{m}$ is an abelian ideal of $\mathbf{g}$. By Proposition 4.2 , if $\bar{m} \subset m$, then $\bar{m}=m^{\prime}$. Thus, it is sufficient to show that $d \sigma(X)=-X$, for $X \in \bar{m}$, where $\sigma$ is the involutive automorphism of $G$. Since $G^{\prime}$ has Lie subalgebra $\mathrm{m}^{\prime}$ which is contained in $\mathrm{m}$, we have $\sigma(g)=g^{-1}$, for $g \in G^{\prime}$. Now $\bar{G}^{\prime}$ being the closure of $G^{\prime}$ in $G$, we also have $\sigma(g)=g^{-1}$, for $g \in \bar{G}^{\prime}$. Consequently, we have $d \sigma(X)=-X$, for $X \in \bar{m}$. This proves the lemma. Q.E.D.

If $H^{\prime}=G^{\prime} \cap H, \sigma^{\prime}=\sigma \mid G$, then from the lemma above, $\left(G^{\prime}, H^{\prime}, \sigma^{\prime}\right)$ is a closed normal symmetric subspace of $(G, H, \sigma)$. If we put $G^{*}=G / G^{\prime}, H^{*}=$ $H / H^{\prime}$, and define $\sigma^{*}$ to be the involutive auotmorphism of $G^{*}$ induced by $\sigma$, then $\left(G^{*}, H^{*}, \sigma^{*}\right)$ is a symmetric space, and moreover, $M$ is a fibre space over the affine symmetric space $G^{*} / H^{*}$ with fibre $G^{\prime} / H^{\prime}$ in a natural manner. Summing up we have the following.

THEOREM 4.6. Let $M=G / H$ be an affine symmetric space. Then $M$ is a 
fibre bundle over $G^{*} / H^{*}$ with fibre isomorphic to $G^{\prime} / H^{\prime}$. Moreover, the base manifold has trivial nullity distribution.

Proof. It remains to show that $G^{*} / H^{*}$ has zero nullity distribution. We first note that $H^{\prime}=H \cap G^{\prime}$ is a discrete group, since $m^{\prime} \cap \mathfrak{h}=0$. Hence, the Lie algebra $g^{*}$ of $G^{*}$ is equal to $\mathfrak{m}^{*}$, where $\mathrm{m}^{*}=\mathrm{m} / \mathrm{m}^{\prime}$. We identify $\mathrm{m}^{*}$ with the tangent space at $\overline{0}=e H^{*} \in G^{*} / H^{*}$. It is sufficient to show that $G^{*} / H^{*}$ has zero nullity space $N_{\overline{0}}$ at $\overline{0}$ with respect to the unique $G^{*}$-invariant connection in $G^{*} / H^{*}$. Let $\bar{X} \in N_{\overline{0}} \subset \mathrm{m}^{*}$. Since $G^{*}$ acts effectively on $G^{*} / H^{*}$, it follows from Proposition 4.2 that $\left[\bar{X}, m^{*}\right]=0$. Let $X \in m$ represent $\bar{X}$ in $m^{*}$. Then $[X, m] \subseteq m^{\prime}$, which implies that $[X, m] \subseteq \mathfrak{h} \cap \mathrm{m}^{\prime}=0$. Hence, we have $X \in N_{0}$ $=m^{\prime}$. This proves that $\bar{X}=0$. Q.E.D.

COROLlary 4.7. The fibres of the above bundle are flat and totally geodesic submanifolds of $M$.

ProoF. Follows immediately from Theorem 3.5.

5. Riemannian symmetric spaces. Let $(M, g)$ be a Riemannian symmetric space and $\nabla$ the corresponding Riemannian connection with respect to the metric g. A Riemannian symmetric space is associated with a symmetric space $(G, H, \sigma)$ where $G$ is the identity component of the Lie group of all isometries on $M$, and $H$ here is the compact subgroup of $G$ which leaves a point $0 \in M$ fixed. We recalled that $(M, g)$ is said to be of compact type (respectively noncompact type) if $G$ is compact (respectively noncompact) and semisimple. It is said to be of Euclidean type if $m$ is an abelian ideal in $g$, where $g=\mathfrak{b}+\mathfrak{m}$ is such that $\mathfrak{b}$ is the Lie algebra of $H$, and $m$ is the eigenspace of $d \sigma$ for the eigenvalue -1 .

Proposition 5.1. Let $(M, g)$ be a Riemannian symmetric space of the compact or noncompact type. Then $(M, g)$ has zero nullity distribution.

Proof. We have from Proposition 4.2 that $\mathrm{m}^{\prime}=N_{0}$ is an abelian ideal of g. Since $g$ is semisimple, $\mathrm{m}^{\prime}$ equals zero. The proposition now follows since $N_{x} \cong N_{0}$ for all $x \in M$. Q.E.D.

Proposition 5.2. Let $(M, g)$ be a simply connected Riemannian symmetric space. Then $M$ is a product, $M=M_{0} \times M_{-} \times M_{+}$, where $M_{0}$ is a Euclidean space, $M_{-}$and $M_{+}$are Riemannian symmetric spaces of the compact and noncompact type, respectively.

Proof. See [2, p. 208].

It is clear that the nullity distribution $N$ of a Riemannian symmetric space $(M, g)$ of Euclidean type is equal to the whole tangent bundle TM. Thus, it follows from Proposition 5.1 that the nullity distribution $N$ of a simply connected Riemannian symmetric space $(M, g)$ is just the Euclidean part of the manifold as in Proposition 5.2. If we compare Theorem 4.6 and Proposition 5.2, we see that 
in the affine symmetric space (we do not make the assumption that it is simply connected), we can also 'factor' out the flat part of the manifold, and the remaining part has zero nullity distribution.

6. An application. Let $G$ be a compact Lie group, and $B$ be an ad $G$-invariant positive definite symmetric bilinear form on the Lie algebra 8 of the Lie group $G$. Then $B$ induces a $G$-bi-invariant Riemannian metric $g$ on $G$. Let $\nabla$ be the unique Riemannian connection of $(G, g)$. It is clear that $\nabla$ is bi-invariant and for $X, Y \in g$, we have $\nabla_{X} Y=1 / 2[X, Y]$ and $R(X, Y)=-1 / 4$ ad $[X, Y]$ (see [4, Vol. II, p. 204]).

Since $\nabla$ is $G$-bi-invariant, the nullity distribution $N$ of the Lie group $G$ is biinvariant. Hence $N$ gives a regular distribution on $G$. Moreover, considered as a bundle over $G$, it is trivial.

Proposition 6.1. The compact Lie group is semisimple if and only if the nullity distribution is zero.

Proof. It is easily shown that the nullity at identity $N_{e}$ is equal to zero. By virtue of the invariant property of $N$, we have $N=0$.

Conversely, if $G$ is not semisimple, then we have a nonzero centre 8 of 8 . Therefore, there exists $X \neq 0$ in 8 such that $[X, Y]=0$ for all $Y \in 8$. Thus $\operatorname{ad}[X, Y]=0$ for all $Y \in 8$. Consequently, $N_{e}$ contains the nonzero vector $X$, and this implies that $N$ is nonzero. Q.E.D.

Corollary 6.2. Let $G$ be a compact Lie group. Then $N_{e}$ is the centre of 8 .

Proof. Follows immediately from above proposition.

Proposition 6.1 gives another geometric characterization of semisimple Lie groups. It says that if the compact Lie group has 'flat' leaves with respect to any bi-invariant metric, then it is not semisimple.

7; Compact Lie group actions. Let $F: G \times M \rightarrow M$ be an action of a compact connected Lie group on a manifold $M$. Every point $m \in M$ determines the map $A_{m}: G \rightarrow M$ given by $A_{m} a=a m$, for $a \in G$ and $m \in M$. Throughout this section, we assume that $G$ acts almost freely on $M$ so that Image $\left(d A_{m}\right)_{e} \subset$ $T M$ has the same dimension for all $m \in M$. These subspaces define a distribution $E$ on the manifold $M$.

Proposition 7.1. The distribution $E$ is integrable. Moreover, $E$ is trivial.

Proof. The $C(M)$-module $\Gamma(E)$ is generated by the fundamental vector fields $X^{*}$, where

$$
X_{m}^{*}=d A_{m}(X), \quad \text { for } X \in \mathfrak{g} \text { and } m \in M
$$


Since $\left[X^{*}, Y^{*}\right]=[X, Y]^{*}$, for $X, Y \in \mathbb{g}$, it follows that $E$ is integrable. The distribution $E$ is trivial because $E \cong M \times$ 8. Q.E.D.

Since $G$ is compact, it induces a Riemannian metric $g$ on $M$. Moreover $g$ can be chosen such that $G$ acts as a group of isometries. With respect to $g$, let $Q$ be the orthogonal complement of $E$ so that $T M=E \oplus Q$. It is clear that both $E$ and $Q$ are both $G$-invariant, and the metrics induced on $E$ and $Q$ are also $G$ invariant.

Next, we want to construct a metric connection $\bar{\nabla}$ on $Q$ such that the curvature $\bar{R}$ satisfies $\bar{R}(X,-)=0$ for $X \in \Gamma(E)$. We can do this by first constructing a $G$-invariant metric connection $D$ on $Q$. Let $D^{\prime}$ be any metric connection on $Q$, and set

$$
D_{X} Z=\int_{G} a^{-1}\left(D_{a X}^{\prime}(a Z)\right) d a, \quad \text { for } X \in T M_{m} \text { and } Z \in \Gamma(Q) \text {. }
$$

Then it is clear that $D$ remains a metric connection, and becomes $G$-invariant; that is

$$
D_{a} Y^{a Z}=a D_{X} Z, \quad \text { for } Y \in \mathfrak{X}(M), Z \in \Gamma(Q) \text { and } a \in G .
$$

Differentiating the above equation, we have

$$
D_{\left[X^{*}, Y\right]} Z+D_{Y}\left[X^{*}, Z\right]=\left[X^{*}, D_{Y} Z\right] \text {, for } X \in \mathbb{g}, Y \in \mathfrak{X}(M) \text { and } Z \in \Gamma(Q) \text {. }
$$

Let $i, j, p$ and $\pi$ be the natural maps of the following split exact sequence

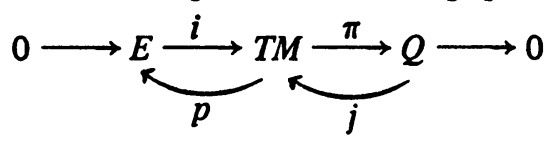

We now can construct the required metric connection $\bar{\nabla}$ on $Q$ by setting

$$
\bar{\nabla}_{X} Y= \begin{cases}D_{X} Y, & \text { for } X, Y \in \Gamma(Q), \\ \pi[X, Y], & \text { for } X \in \Gamma(E) \text { and } Y \in \Gamma(Q) .\end{cases}
$$

It is clear that $\bar{\nabla}$ extends to a connection on $Q$.

Proposition 7.2. The connection $\bar{\nabla}$ on $Q$ is a metric connection. Moreover, the curvature $\bar{R}$ satisfies

$$
\bar{R}(X,-)=0 \text { for } X \in \Gamma(E) .
$$

Proof. The first part of the proof is similar to $[7,86]$ and follows easily from the above construction. To prove the second statement, we first show that

$$
\bar{R}(X, Y)=0, \text { for } X, Y \in \Gamma(E) \text {. }
$$

In fact, fix $W \in \Gamma(Q)$, and define $Z \in \Gamma(E)$ by

$$
\bar{\nabla}_{Y} W=[Y, W]+Z \text {. }
$$


Since $E$ is integrable, $[X, Z] \in \Gamma(E)$. Hence $\pi[X, Z]=0$, and so

$$
\bar{\nabla}_{X} \bar{\nabla}_{Y} W=\pi([X,[Y, W]]) .
$$

From this and the Jacobi identity, it follows that

$$
\begin{aligned}
\bar{R}(X, Y) W & =\bar{\nabla}_{X} \bar{\nabla}_{Y} W-\bar{\nabla}_{Y} \bar{\nabla}_{X} W-\bar{\nabla}_{[X, Y]} W \\
& =\pi\{[X,[Y, W]]-[Y,[X, W]]-[[X, Y], W]\} \\
& =0 .
\end{aligned}
$$

Hence, we have $R(X, Y)=0$, for $X, Y \in \Gamma(E)$. Consequently, to show (7.2), it is sufficient to show that

$$
\bar{R}(X, Y)=0, \text { for } X \in \Gamma(E), Y \in \Gamma(Q) \text {. }
$$

Since the fundamental vector fields span the fibres of $E$, it is sufficient to show that

$$
\bar{R}\left(X^{*}, Y\right)=0 \quad \text { for } Y \in \Gamma(Q), X \in \boldsymbol{8}
$$

But for $W \in \Gamma(Q)$,

$$
\bar{R}\left(X^{*}, Y\right) W=\pi\left\{\left[X^{*}, D_{Y} W\right]-D_{Y}\left[X^{*}, W\right]-D_{\left[X^{*}, Y\right]} W\right\} .
$$

Since $g$ is $G$-invariant, we see that $\left[X^{*}, Z\right] \in \Gamma(Q)$, for $Z \in \Gamma(Q)$. Therefore, (7.3) becomes

$$
\bar{R}\left(X^{*}, Y\right) W=\left[X^{*}, D_{Y} W\right]-D_{Y}\left[X^{*}, W\right]-D_{\left[X^{*}, Y\right]} W=0 .
$$

This completes the proof of the proposition. Q.E.D.

THEOREM 7.3. Given an almost free action of a compact Lie group $G$ on $M$, then there exists an affine structure on $M$ with nullity index at least the dimension of $G$.

Proof. Let us first prove a lemma.

Lemma 7.4. Let $F=E \oplus Q$, and let $D$ (respectively $D^{\prime}$ ) be a connection on $E$ (respectively on $Q$ ). We define $\nabla$ as follows:

$$
\nabla_{X} W=D_{X}(p W)+D_{X}^{\prime}(\pi W), \text { for } X \in \mathfrak{X}(M) \text { and } W \in \Gamma(F) \text {, }
$$

where $p, \pi$ are as in (7.1). Then $\nabla$ is a connection on $F$. Moreover, the curvature $R$ of $\nabla$ satisfies

$$
R(X, Y) W=k(X, Y) p W+k^{\prime}(X, Y) \pi W,
$$

where $k, k^{\prime}$ denote the curvature of $D, D^{\prime}$ respectively. 
Proof. It follows from routine verification.

Proof of THEOREM. Since $E$ is trivial, there exists a flat metric connection $D$ on $E$. From the preceding lemma, $D$ and the connection $\bar{\nabla}$ in Proposition 7.2 define a connection $\nabla$ on TM. It is easy to verify that $\nabla$ is metric. From Lemma 7.4, we have

$$
R(X, Y) Z=k(X, Y)(p Z)+\bar{R}(X, Y)(\pi Z), \quad \text { for } X, Y, Z \in \mathfrak{X}(M) .
$$

Since $D$ is flat, we therefore have

$$
R(X, Y) Z=\bar{R}(X, Y)(\pi Z) .
$$

Suppose $X \in \Gamma(E)$, then, from Proposition 7.2, we have

$$
R(X, Y) Z=\bar{R}(X, Y)(\pi Z)=0 .
$$

This shows that $E \subset N$. Hence this completes the proof. Q.E.D.

8. Partial integrability. A $G$-structure on a manifold $M$ is called integrable (locally flat) if it is locally equivalent to the canonical flat $G$-structure. Quite often, the integrability of a certain $G$-structure can be expressed by the vanishing of certain tensors on $M$. The following are a few examples.

\section{$G$-structure}

(1) Affine or Riemannian structure

(2) Almost complex structure

(3) Conformal structure tensor

Curvature tensor

Nijenhuis torsion

Conformal tensor

We can define nullity distributions for those $G$-structures characterized by tensors. If the resulting nullity distribution is involutive, then we can use the theory developed to study the resulting foliated structure on the manifold $M$.

In the remainder of this section, we devote ourselves to studying the partial integrability of an almost complex manifold. Let $M$ be an almost complex manifold with almost complex structure $J$. The Nijenhuis torsion of the almost complex manifold $M$ can be regarded as the map $T: T M \rightarrow \operatorname{Hom}(T M, T M)$. Then the nullity distribution $N$ of the almost complex manifold $M$ is defined by setting $N=$ Kernel $T$.

A connection $\nabla$ on the almost complex manifold $M$ is said to be almost complex if the almost complex structure $J$ is parallel with respect to $\nabla$, that is $\nabla_{X}(J Y)=J \nabla_{X} Y$ for all $X, Y \in \mathfrak{X}(M)$. Every almost complex manifold $M$ admits an almost complex connection such that its torsion $T^{\prime}$ is given by $T=8 T^{\prime}$ (see [4, Vol. II, p. 143]). The following proposition summarizes some basic properties of $N$.

Proposition 8.1. (i) The nullity index $\mu(x)$ is upper semicontinuous. 
(ii) The nullity distribution $N$ is invariant under the endomorphism $\mathrm{J}$.

(iii) If $T$ is parallel with respect to the almost complex connection $\nabla$, then $N$ is involutive.

Proof. Statement (i) is proved just like Proposition 1.1. To show (ii), let $X \in N_{x}$. We have

$$
\begin{aligned}
T(J X, Y) & =-T(J J X, J Y), \quad \text { for } Y \in T M_{x}, \\
& =T(X, J Y)=0
\end{aligned}
$$

which implies that $J X \in N_{x}$. This proves (ii). To prove (iii), let $X, Y \in \Gamma(U, N)$. Then

$$
0=T(X, Y)=8 T^{\prime}(X, Y)=8\left(\nabla_{X} Y-\nabla_{Y} X-[X, Y]\right) .
$$

Thus $[X, Y]=\nabla_{X} Y-\nabla_{Y} X$. Therefore, for all $Z \in \Gamma(U, T M)$, we have

$$
T([X, Y], Z)=T\left(\nabla_{X} Y-\nabla_{Y} X, Z\right)=T\left(\nabla_{X} Y, Z\right)-T\left(\nabla_{Y} X, Z\right) .
$$

Since $T$ is parallel, we have

$$
0=\left(\nabla_{X} T\right)(Y, Z)=\nabla_{X} T(Y, Z)-T\left(\nabla_{X} Y, Z\right)-T\left(Y, \nabla_{X} Z\right)=-T\left(\nabla_{X} Y, Z\right)
$$

since $X, Y \in \Gamma(U, N)$. Hence, $N\left(\nabla_{X} Y, Z\right)=0$. Similarly, we can show that $N\left(\nabla_{Y} X, Z\right)=0$. This shows that $T([X, Y], Z)=0$ for all $Z \in \Gamma(U, T M)$, which implies that $[X, Y] \in \Gamma(U, N)$. This completes the proof. Q.E.D.

THEOREM 8.2. If $N$ is nonsingular, then the leaves of the nullity distribution of the almost complex manifold $M$ are complex manifolds which are totally geodesic in $M$.

Proof. Let $L$ be a leaf of $N$. The almost complex structure $J$ of $M$ induces an almost complex structure on $L$ (Proposition 8.1(ii)). Moreover, the Nijenhuis torsion of the almost complex manifold $L$ vanishes. Hence $L$ is a complex manifold. From the proof of the above proposition, we see that $\nabla_{X} Y \in N$ for $X, Y \in$ $N$. As in $\S 3$, we conclude that $L$ is auto-parallel, which implies that $L$ is totally geodesic. Q.E.D.

\section{REFERENCES}

1. S. S. Chern and N. H. Kuiper, Some theorems on the isometric imbedding of compact Riemann manifolds in Euclidean space, Ann. of Math. (2) 56 (1952), 422-430. MR 14, 408.

2. S. Helgason, Differential geometry and symmetric spaces, Academic Press, New York and London, 1962. MR 26 \#2986.

3. F. Kamber and $\mathrm{Ph}$. Tondeur, Characteristic invariants of foliated bundles, Manuscripta Math. 11 (1974), 51-89. MR 48 \#12556.

4. S. Kobayashi and K. Nomizu, Foundations of differential geometry. Vols. I, II, Wiley, New York and London, 1963. MR 27 \#2945. 
5. R. Maltz, The nullity spaces of the curvature operator, Topologie et Géométrie Différentielle, Vol. 8, Centre Nat. Recherche Sci., Paris, 1966, pp. 1-20. MR 35 \#940.

6. The nullity spaces of curvature-like tensors, J. Differential Geometry 7 (1972), 519-523. MR 48 \#1101.

7. J. S. Pasternack, Topological obstructions to integrability and the Riemannian geometry of smooth foliations, Thesis, Princeton Univ., 1970.

8. S. L. Tan, Nullity and generalized characteristic classes of differentiable manifolds, Thesis, Univ. of Illinois, 1974.

DEPARTMENT OF MATHEMATICS, UNIVERSITY OF MALAYA, KUALA LUMPUR, MALAYSIA 